\title{
Effect of Aerobic Training on Physiological Cost Index and VO2max in Women with Multiple Sclerosis
}

\section{Bayan Fayazi', Abdolhossein Parnow ${ }^{1 *}$, Behroz Ahsan²}

${ }^{1}$ Sports Sciences Faculty, Razi University, Kermanshah, Iran

${ }^{2}$ Department of Brain and Nervous Diseases, Medicine Faculty, Kurdistan University of Medical Sciences, Kurdistan, Iran

\section{A BSTRACT}

Introduction: Multiple Sclerosis (MS) is a chronic central nervous system disorder, which leads to myelin destruction. The purpose of this study was to investigate the effect of aerobic training on physiological cost index and VO2max in women with MS. Materials and Methods: Fifteen women with MS (aged 33.68 \pm 8.22 years) and EDSS $<4$ participated in this study. Participants randomly were divided to experimental group $(n=8)$ and control group $(\mathrm{n}=7)$. An aerobic program of 8 weeks' duration, 3 days/week, $20-40 \mathrm{~min} /$ day, with $60-80 \%$ of HRmax was designed. To measure of physiological cost index, VO2max and additionally, walking endurance of subjects were measured before and after program in both experimental and control groups. Results: Data analysis showed that the aerobic training significantly decreased physiological cost index in experimental group. Additionally, after 8-week aerobic training $\mathrm{VO} 2 \mathrm{max}$ and walking endurance significantly increased in experimental group. Conclusion: Aerobic training can increase walking endurance and improvement physiological cost index in MS diseases and increase VO2max. This indicate the potential for aerobic training in treatment of patients with MS.

\section{Key words:}

1. Exercise

2. Multiple Sclerosis

3. Women

*Corresponding Author: Abdolhossein Parnow

E-mail: parnowabdolhossein@gmail.com 


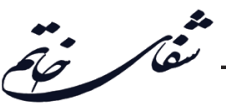

اثر تمرين هوازى بر شاخص هزينهُ فيزيولوزيكى و حداكثر اكسيرن مصرفى در زنان مبتلا به مالتيِل اسكلروز

\author{
بيان فياضى'، عبدالحسين ثرنو'”، بهروز احسن' \\ ' دانشكده علوم ورزشى، دانشكاه رازى، كرمانشاه، ايران \\ 'بخش بيمارى هاى مغز و اعصاب، دانشكده يزشكى، دانشكاه علوم يزشكى كردستان، كردستان، ايران
}

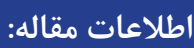

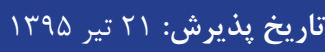

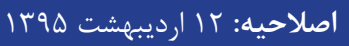

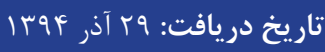

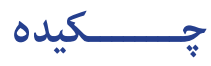

مقدمه: مالتييل اسكلروز يك بيمارى مزمن سيستم عصبى مركزى است كه منجر به تخرئ تخريب ميلين

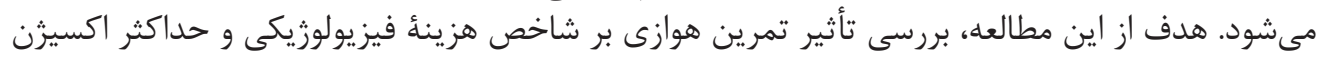

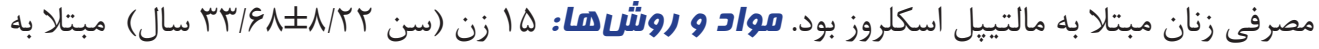

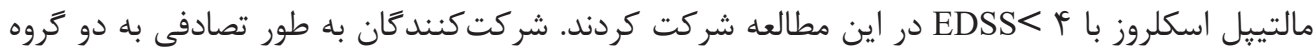

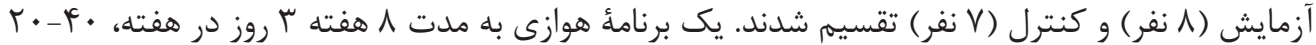

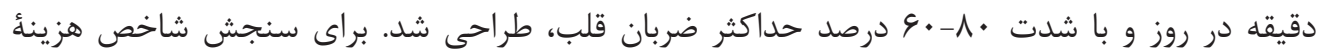

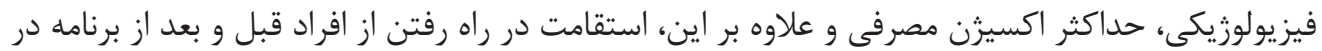

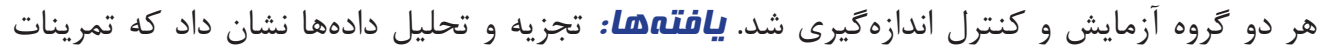

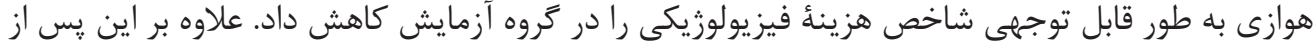

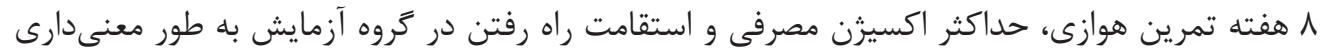

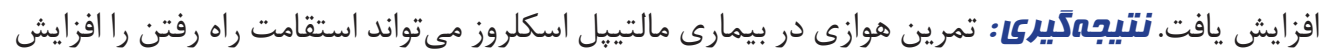

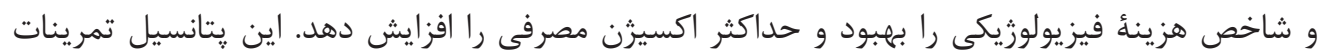

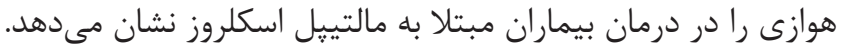

كليد وازمها:

1

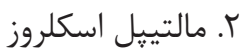
r. 


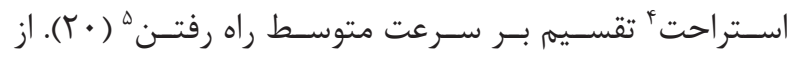

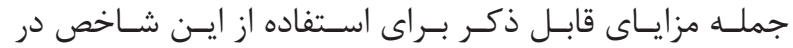

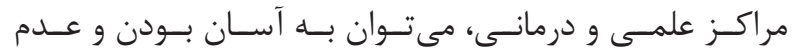

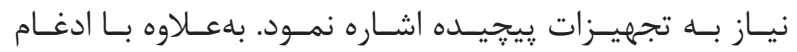

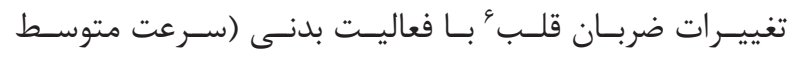

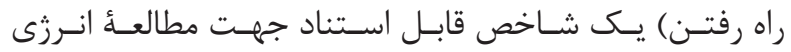

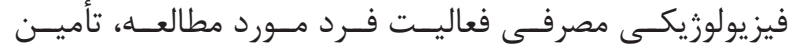

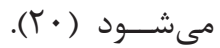

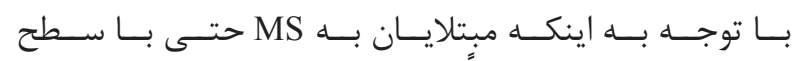

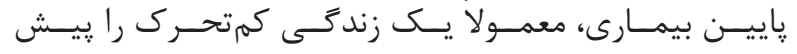

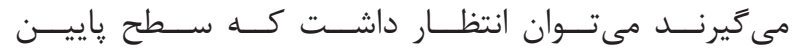

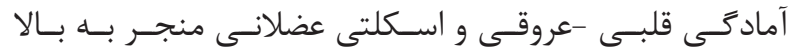

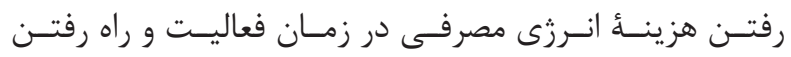

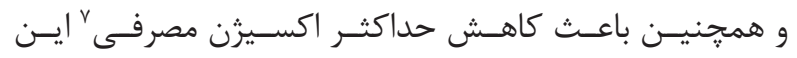

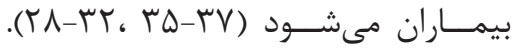

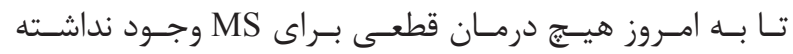

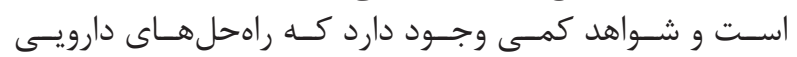

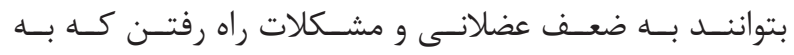

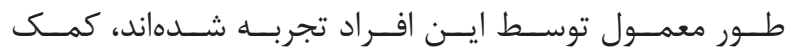

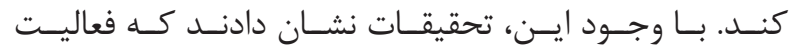

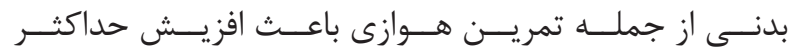

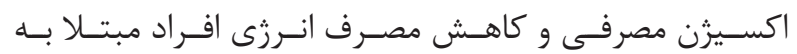

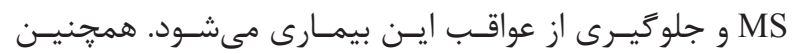

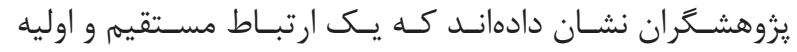

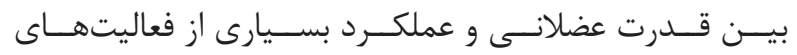

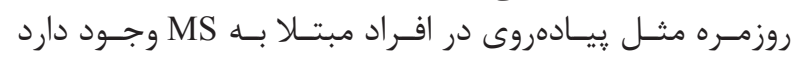

(19. TH. MF, FT)

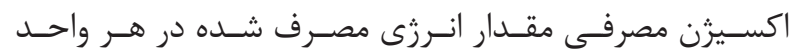

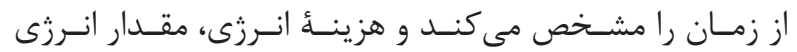

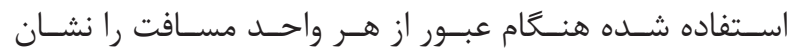

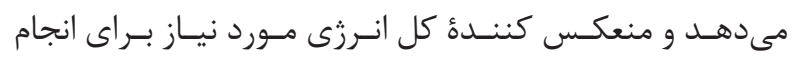

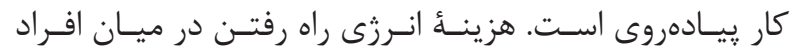

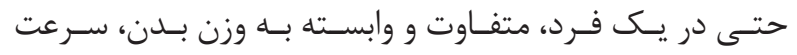

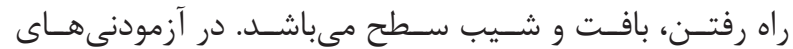

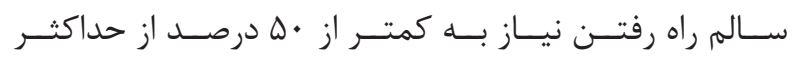

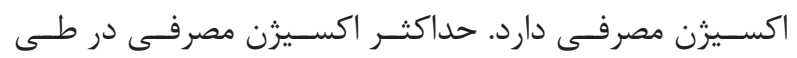

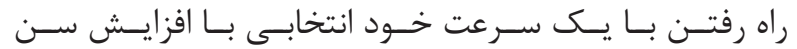

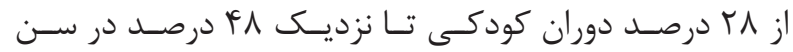

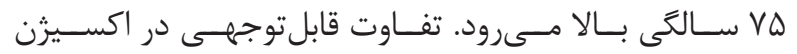

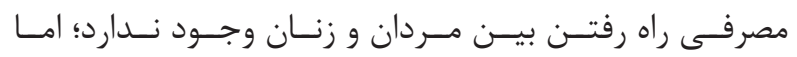

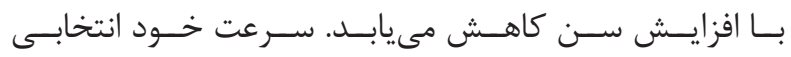

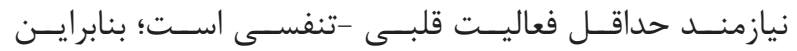

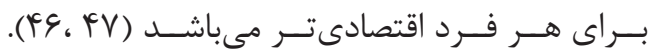

${ }^{1}$ Multiple sclerosis

${ }^{2}$ Physiological cost index

${ }^{3}$ Walking heart rate

${ }^{4}$ Rest heart rate

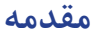

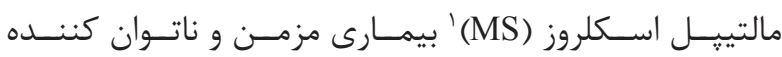

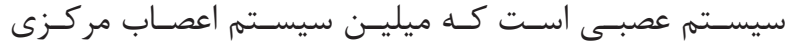

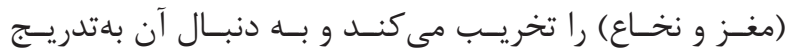

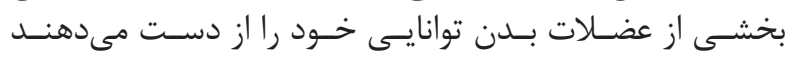

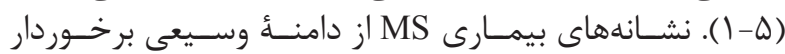

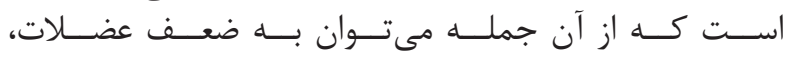

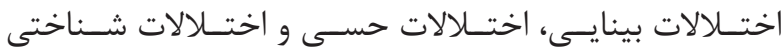

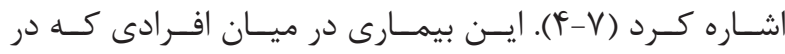

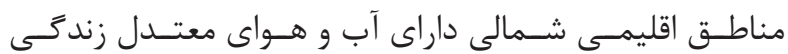

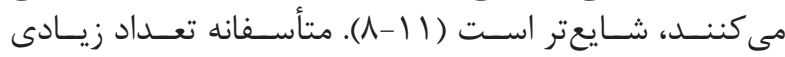

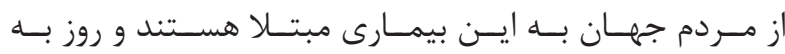

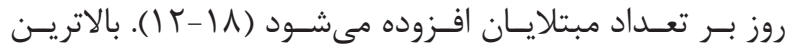

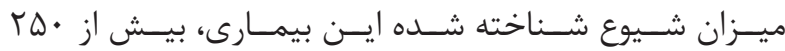

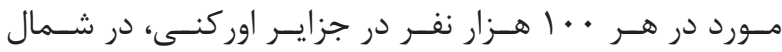

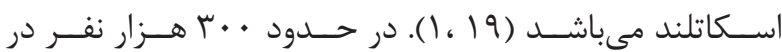

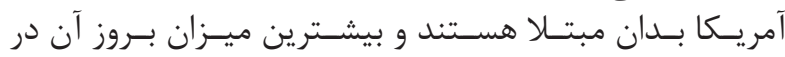

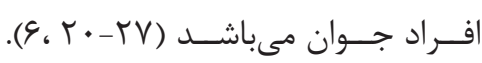

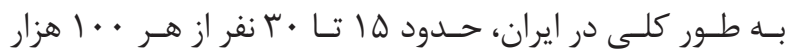

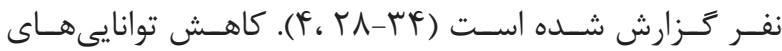

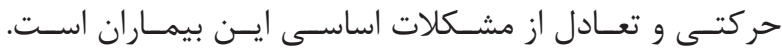

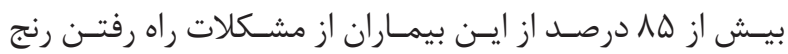

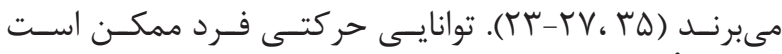

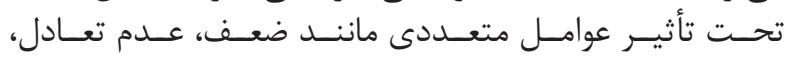

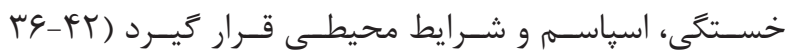

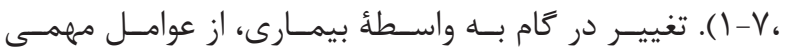

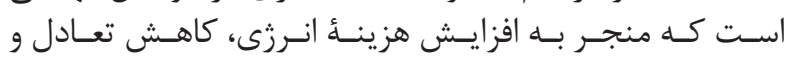

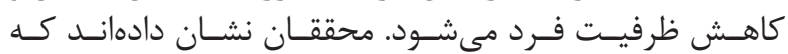

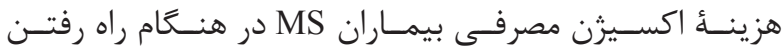

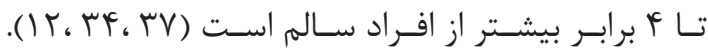

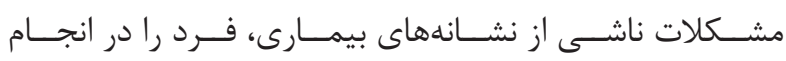

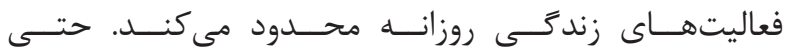

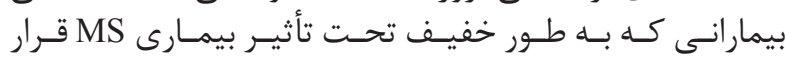

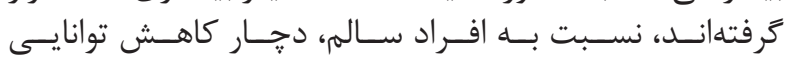

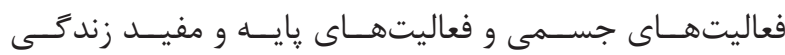

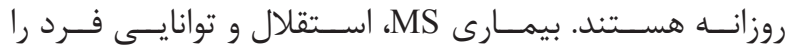

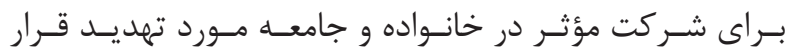

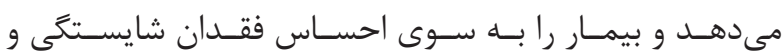

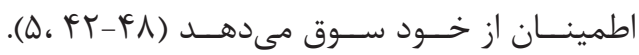

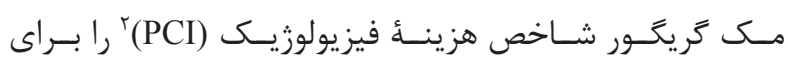

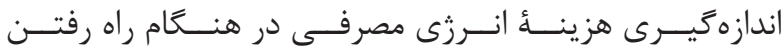

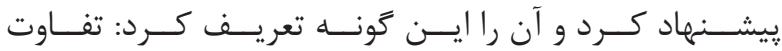

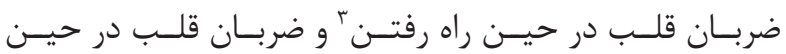

${ }^{5}$ Walking speed

${ }^{6}$ Heart rate

${ }^{7}$ Volume oxygen maximum 


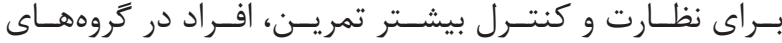

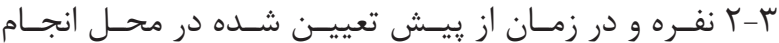

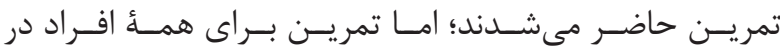

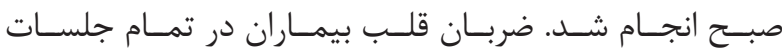

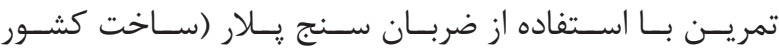

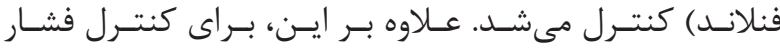

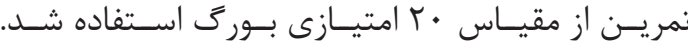

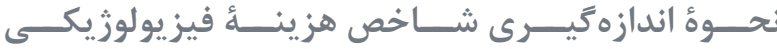

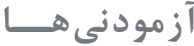

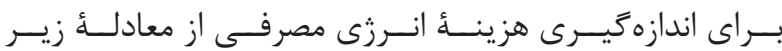

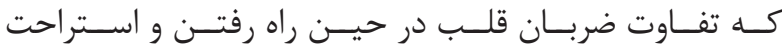

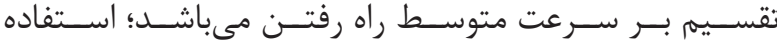

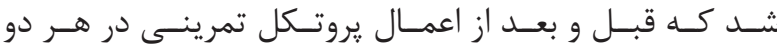

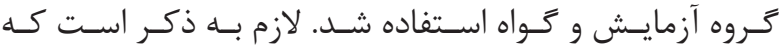

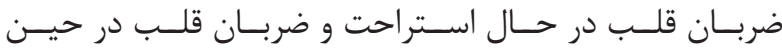

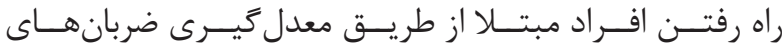

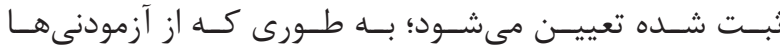

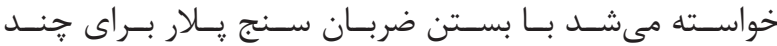

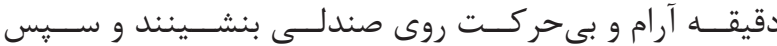

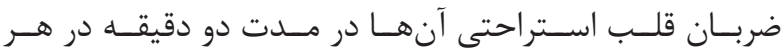

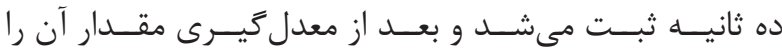

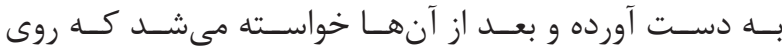

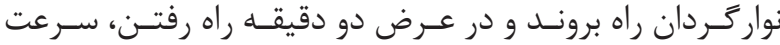

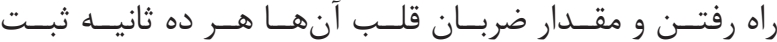

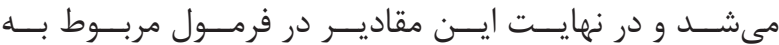

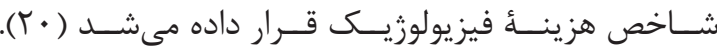

$$
\text { PCI }\left(\frac{\text { baets }}{m}\right)=\frac{W H R\left(\frac{\text { baets }}{\min }\right) R H R\left(\frac{\text { baets }}{\text { min }}\right)}{W S\left(\frac{\mathrm{m}}{\mathrm{min}}\right)}
$$

PCI

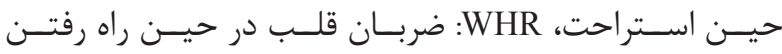

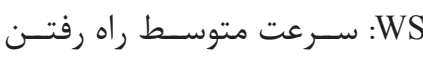

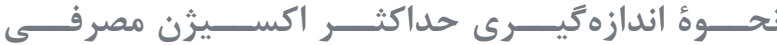

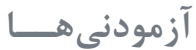

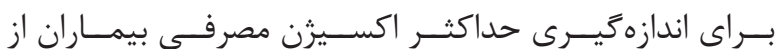

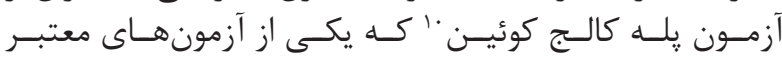

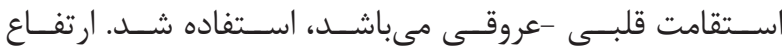

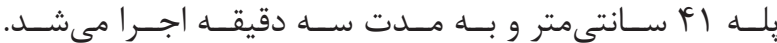

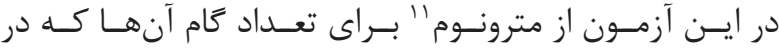

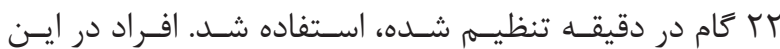

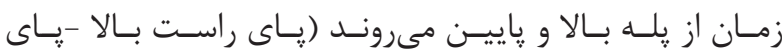

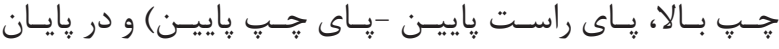

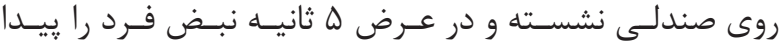

${ }^{8}$ Expanded disability status scale ${ }^{9}$ Neurologist

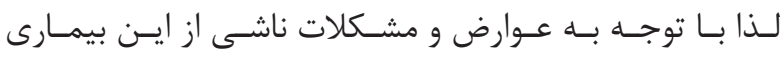

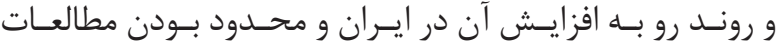

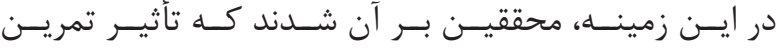

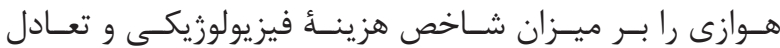

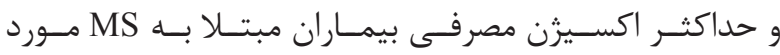

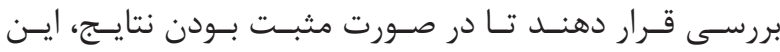

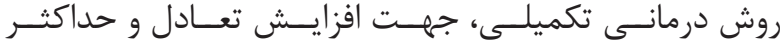

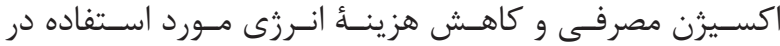

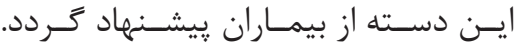

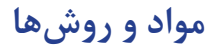

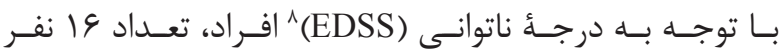

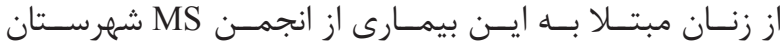

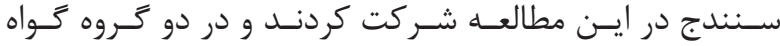

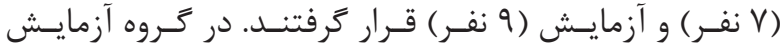

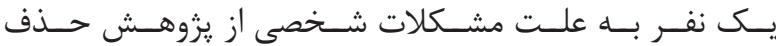

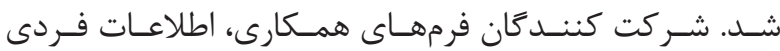

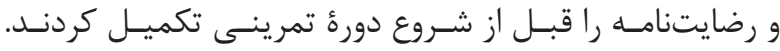

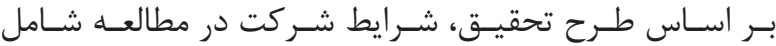

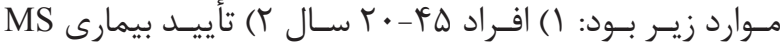

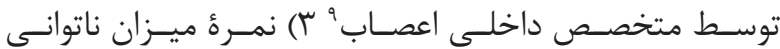

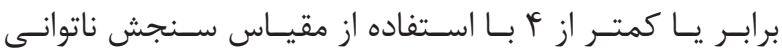

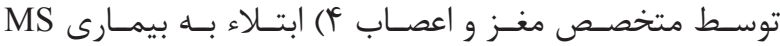

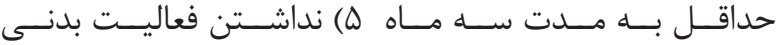

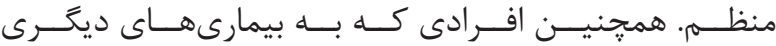

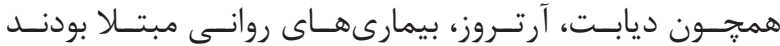

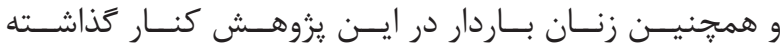

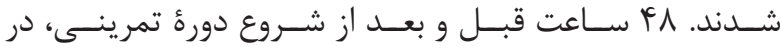

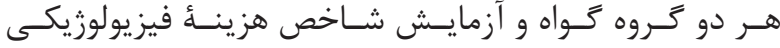

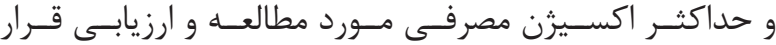

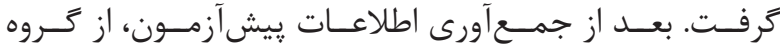

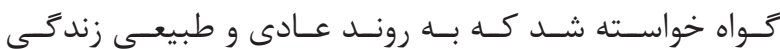

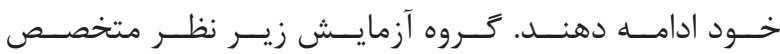

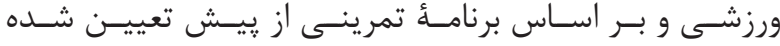

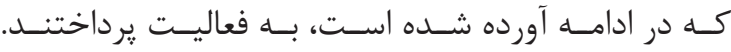

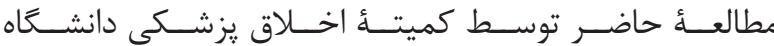

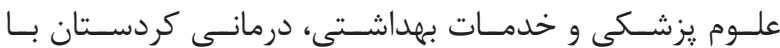
كــ و

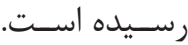
برنامةُ تمرينى تروه آزمايش

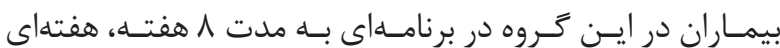

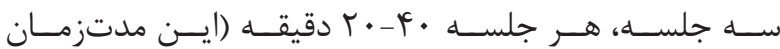

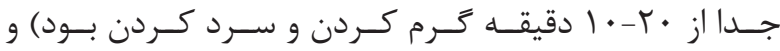

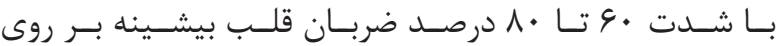

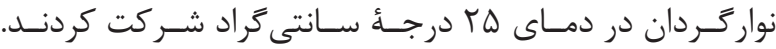

${ }^{10}$ Queen college step test

${ }^{11}$ Metronome 


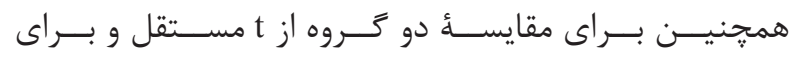

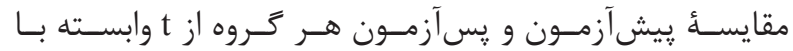

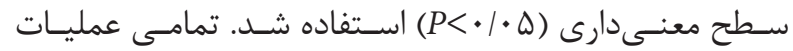

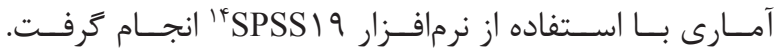

يافتهها

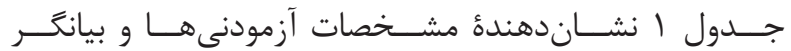

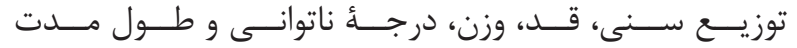

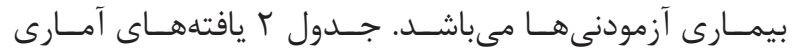

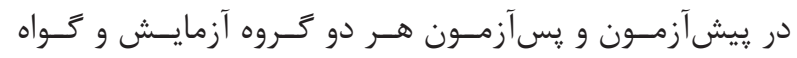

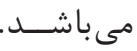

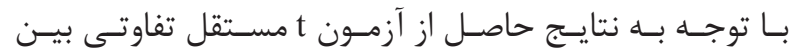

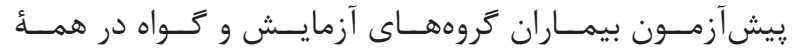

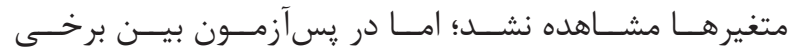

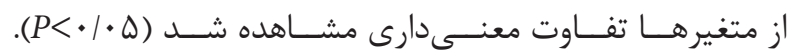

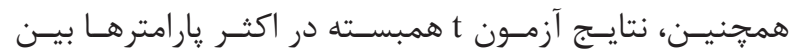

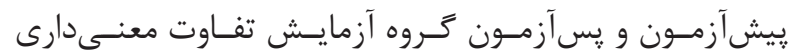

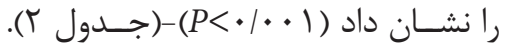

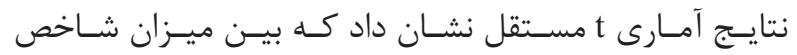

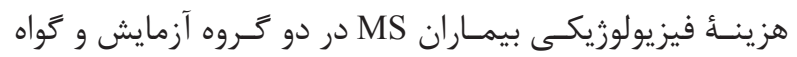

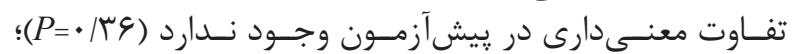

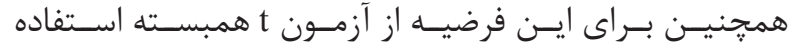

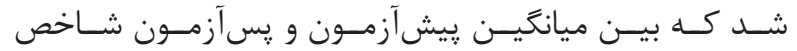

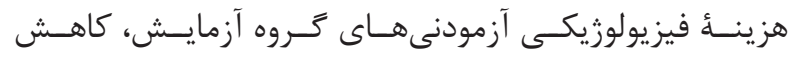

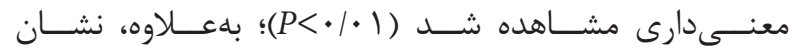

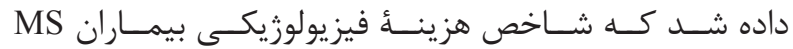

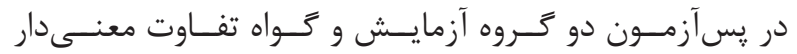

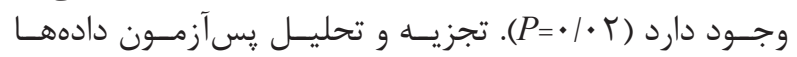

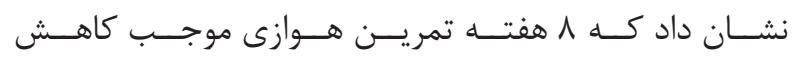

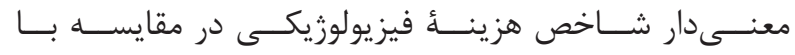

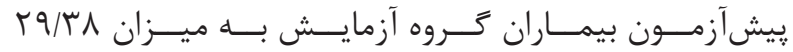

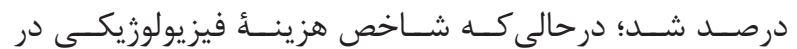

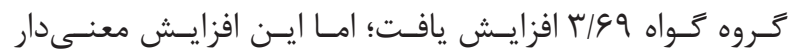

$$
\text { نبـود (نمــودار (1) ) مكواه }
$$

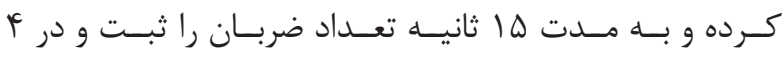

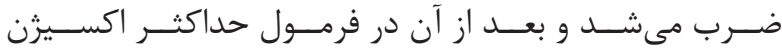

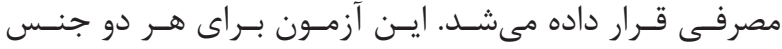

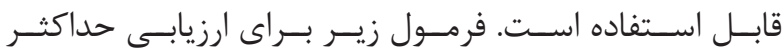

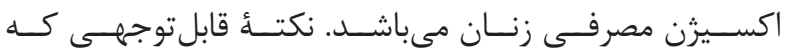

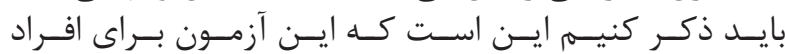

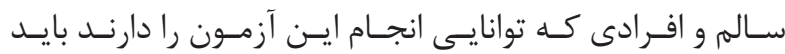

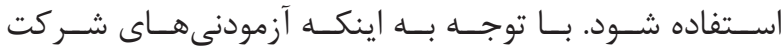

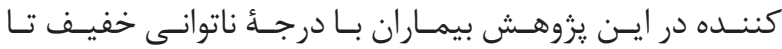

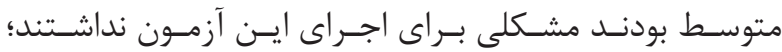

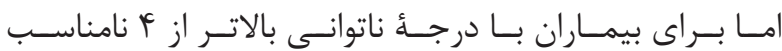

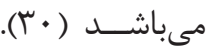

\section{$\left.\mathrm{VO}_{2} \max =\varphi \Delta / \wedge\right)-(\cdot / 1 \wedge \uparrow \vee \times \mathrm{IIR})$}

Vo2max حداكثر اكسيزن مصرفى، HR : ضربان قلب نحوه اندازهَيرى استقامت راه رفتن

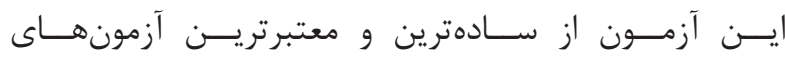

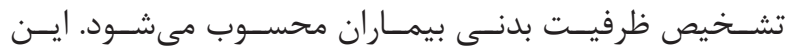

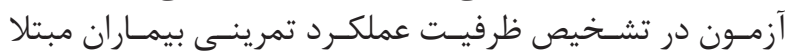

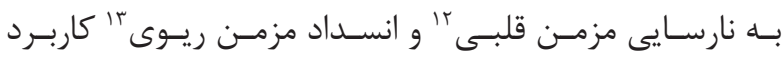

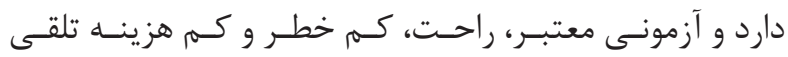

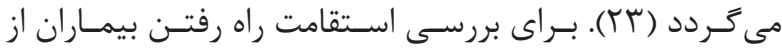

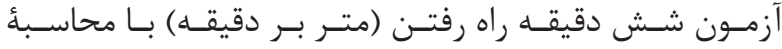

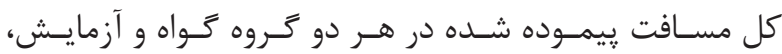

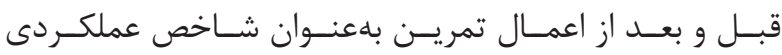

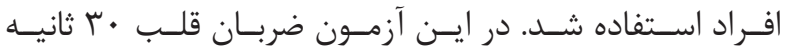

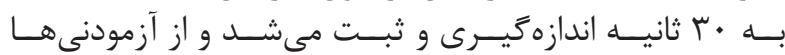

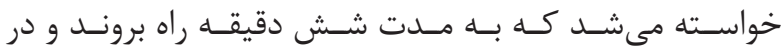

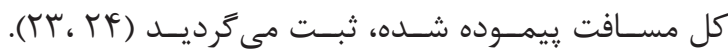

$$
\text { آزمون آمارى }
$$

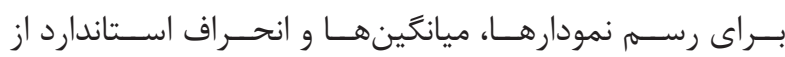

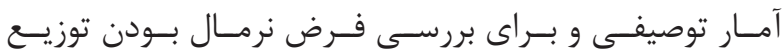

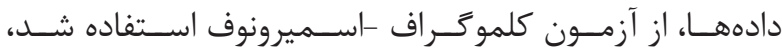

\begin{tabular}{|c|c|c|c|c|c|c|}
\hline بيمارى (سال) مدت & $\begin{array}{c}\text { درجة ناتوانى } \\
\text { (EDSS) } \\
\left(F_{-} \cdot\right)\end{array}$ & وزن (كيلوگرم) & قدانتىمتر ) & (سال) & آزمودنى تعاد & \\
\hline$s / r \pm \Delta / r$. & $1 \pm r$ & $\varepsilon \| /|r \pm \Lambda V / F|$ & س & س & $\Lambda$ & گروه آزمايش \\
\hline 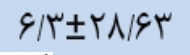 & $T / \cdot \pm \cdot V / \varepsilon V$ & $G T / / T \pm \Lambda \Delta / \Lambda F$ & $|\varepsilon| / F \pm F r / \Lambda \Delta$ & 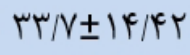 & V & كروه گُواه \\
\hline
\end{tabular}

جدول ا- برخى مشخصات فردى -اجتماعى بيماران مبتلا به MS هر دو گروه گواه و آزمايش شركت كننده در بيشآزمون مطالعه.

${ }^{12}$ Chronic heart failure

${ }^{13}$ Chronic obstructive pulmonary disease

${ }^{14}$ Statistical package for the social sciences 


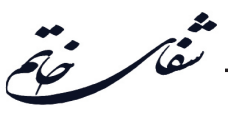

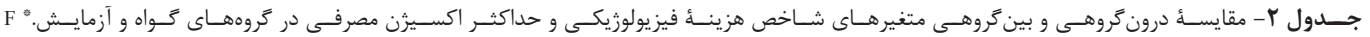

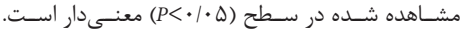

\begin{tabular}{|c|c|c|c|c|c|c|c|c|}
\hline \multicolumn{2}{|c|}{ سطح مسنى دارى } & \multicolumn{3}{|c|}{ كواه } & \multicolumn{3}{|c|}{ آزمايش } & \\
\hline يس آزمون & يَش آزمون & مغئح & يَسآزمون & تيش آزمون & سطئم & جسآزمون & يَيشآزمون & م \\
\hline${ }^{*}+/ \cdots 1$ &.$/ 91$ & $\cdot 1 \cdot v$ & $\Delta r / v / \pm q / \% \Delta$ & $\Delta V / r \Lambda \pm 11 / r r$ & $* *+\cdots 1$ & VNYA $=11 / V \Delta$ & $\Delta V / r r \pm q / r q$ & سرعت متوسط راه رفتن \\
\hline$\cdot / r V$ & $\cdot / \mathrm{V} \wedge$ & $+/ 1 \cdot$ & $\Lambda Y / N \pm N r r$ & $V N \wedge \Delta \pm V / \Delta \Delta$ & $* *+\cdots 1$ & $V r / \Delta \pm N a r$ & $V a \pm D / 1 r$ & ضريان قلي الستراحت \\
\hline${ }^{*}+/ \cdots 1$ & $+/ 90$ & $* \cdot \cdot V$ & $|r \cdot| V| \pm 9| \cdot V$ & $11 N \Delta V \pm \Delta / \Delta \varphi$ & $* *+\cdots 1$ & $111 / \Delta \pm r / r v$ & $11 \mathrm{~N} r v \pm G / r^{r}$ & ضريان قلب در حين راه \\
\hline.$/ 91$ & $\cdot \mid a r$ &.$/ 91$ & $\mathrm{rQ} / \Delta V \pm N / G$ & $\mathrm{ra} / \mathrm{V} / \pm N \wedge \mathrm{G}$ & $\cdot / 40$ & $r a \pm 11 / 9,9$ & $k r \pm 01 / 10$ & 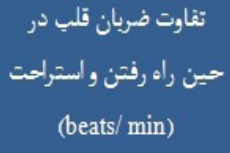 \\
\hline$\oplus+1+r$ & $\cdot /$ te & $\cdot / \mu \psi$ & $\cdot / V \Psi \pm \cdot / r \psi$ & $\cdot / V \cdot \pm \cdot / Y \psi$ & $* * 1+1$ & $\cdot / K a \pm \cdot / 1 Y$ & $\cdot / 9 \mathrm{q} \pm \cdot / 1 \mathrm{~V}$ & فيزيولوريكى (beats/m) فزينه \\
\hline${ }^{*}+\cdots+1$ & $\cdot 1 \cdot 1$ & ${ }^{*}+1+r$ & $r Q / r r \pm \cdot / \Lambda r$ & $r+/ r_{\lambda} \pm 1 / r^{2}$ & $* *+\cdots$ & $|r r|+q \pm r / \varphi q$ & rNADEVIVG & حداكثر اكسيثن مصرفى \\
\hline$*+1+1$ & $\cdot / r v$ & $*+1+4$ & $r \Lambda \% / r \Lambda \pm \Lambda q / r r$ & $r \cdot r / \Delta V \pm \lambda V / q V$ & $* *+\cdots 1$ & $\Delta Q V / Y V \pm \mid Y Y / \cdot 1$ & $f+\Delta V \Delta \pm 1 \cdot T / 1 T$ & $\begin{array}{c}\text { استقامت راه رفتن } \\
\text { (m/min) }\end{array}$ \\
\hline
\end{tabular}

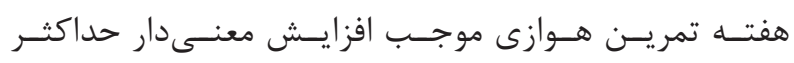

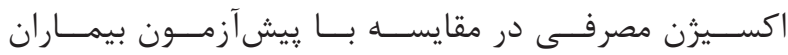

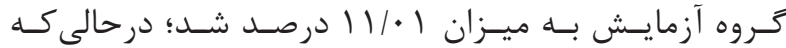

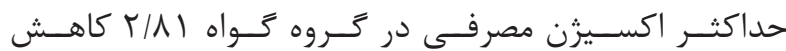

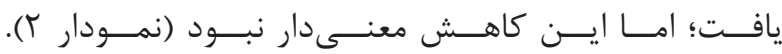

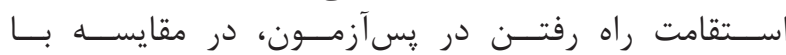

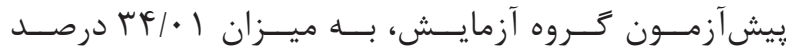

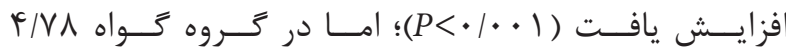

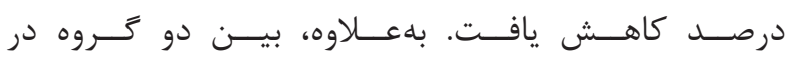

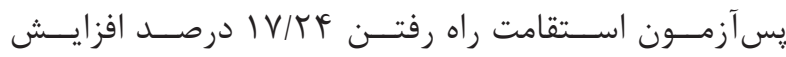

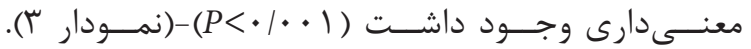

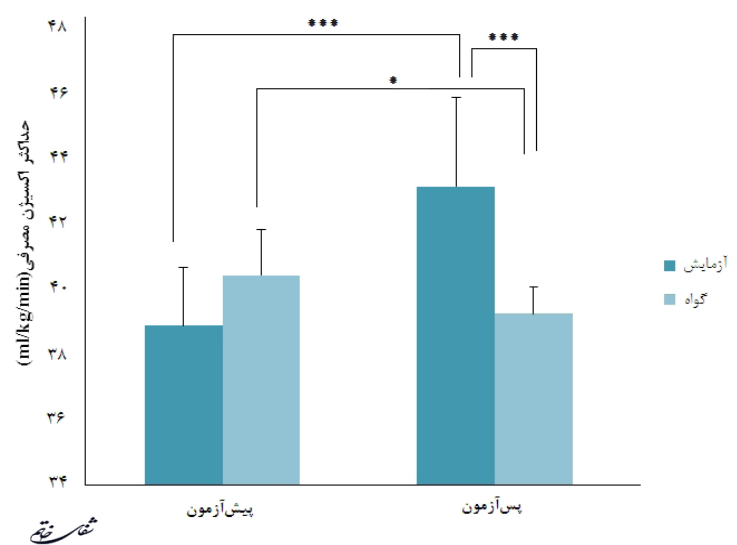

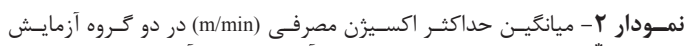

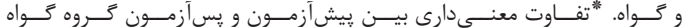

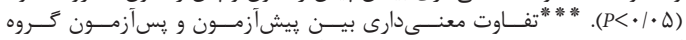

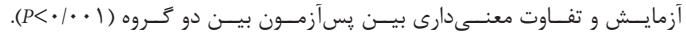

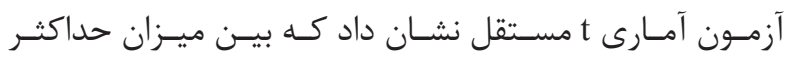

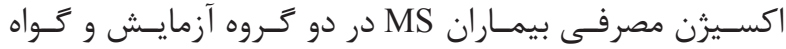

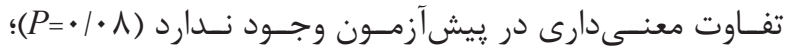

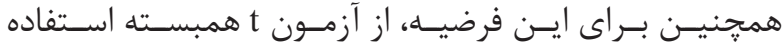

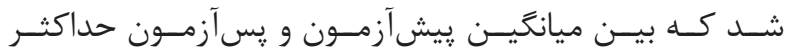

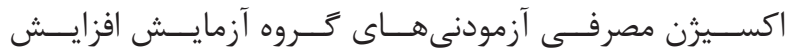

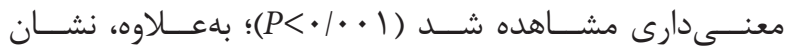

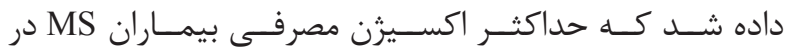

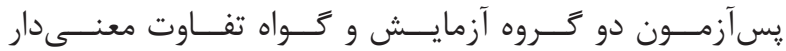

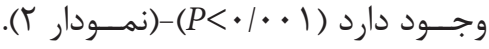

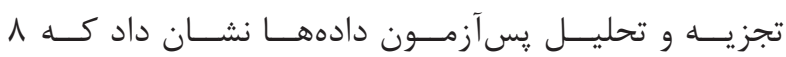

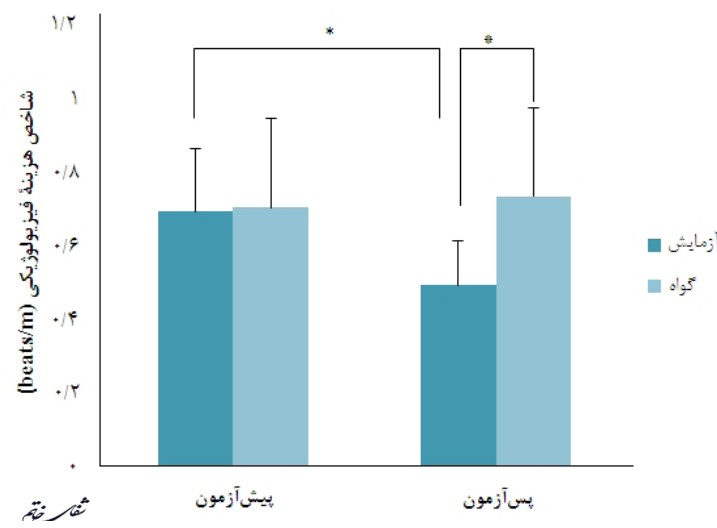

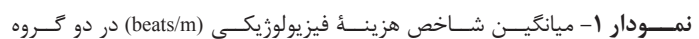

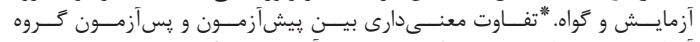

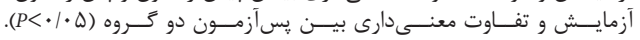




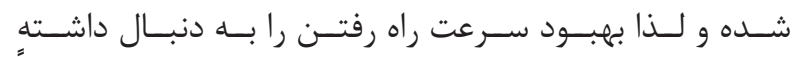

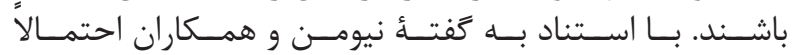

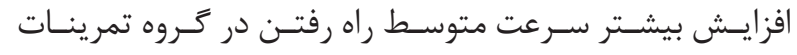

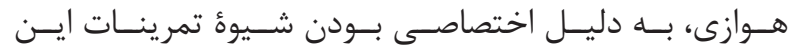

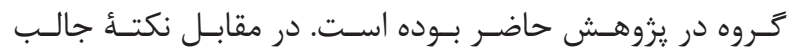

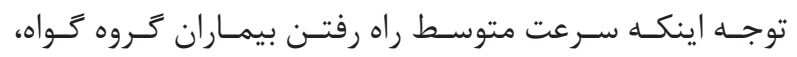

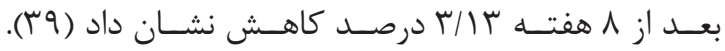

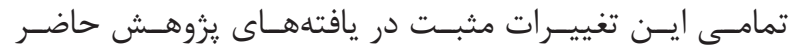

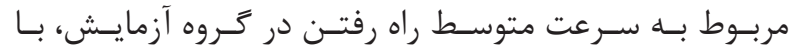

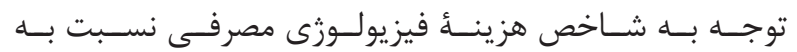

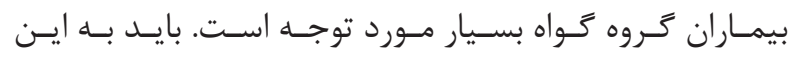

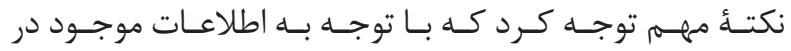

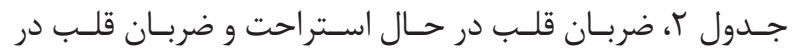

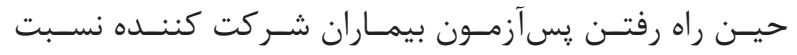

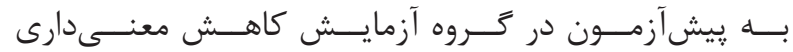

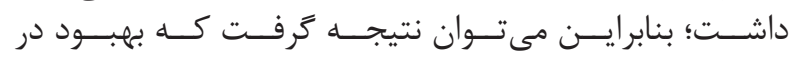

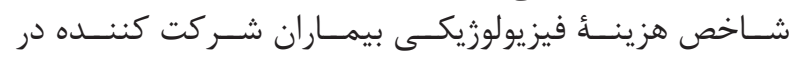

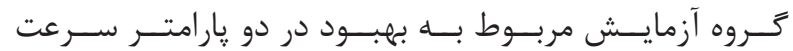

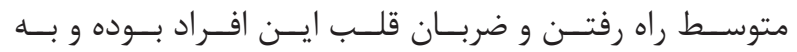

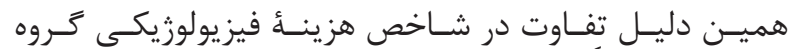

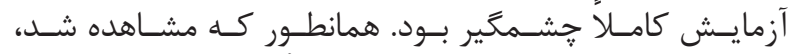

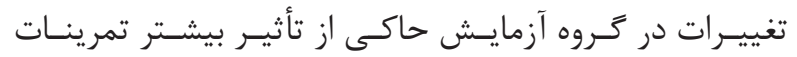

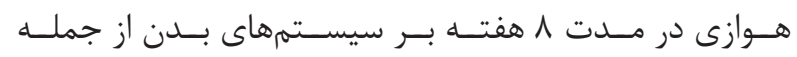

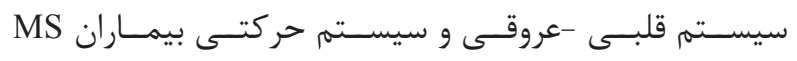
بـوده استـات

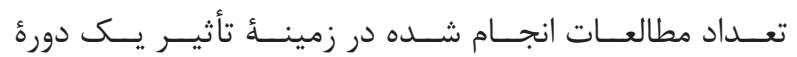

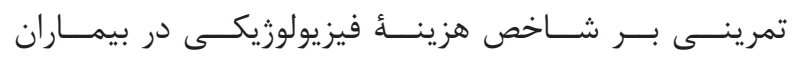

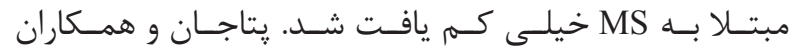

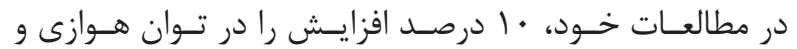

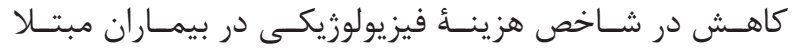

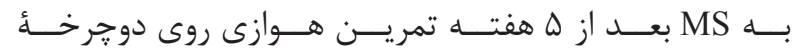

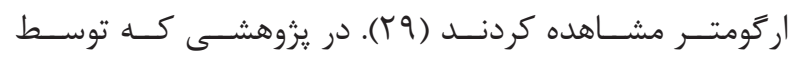

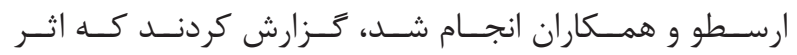

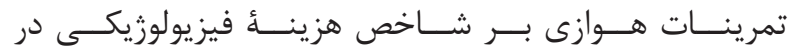

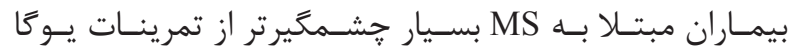

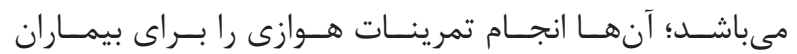

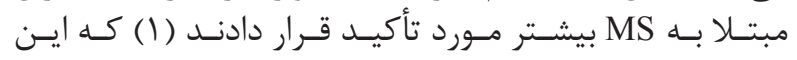

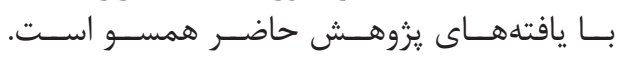

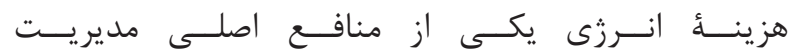

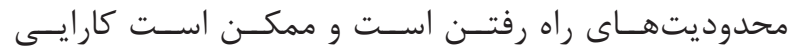

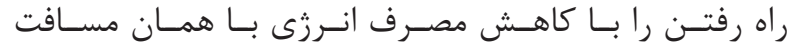

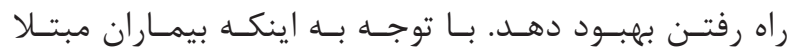

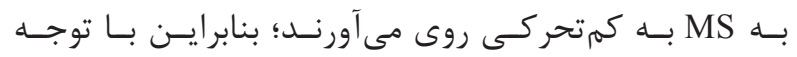

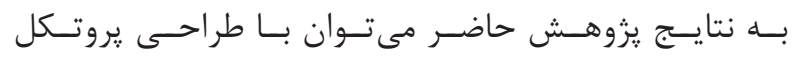

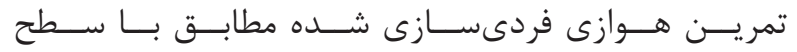

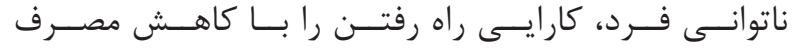

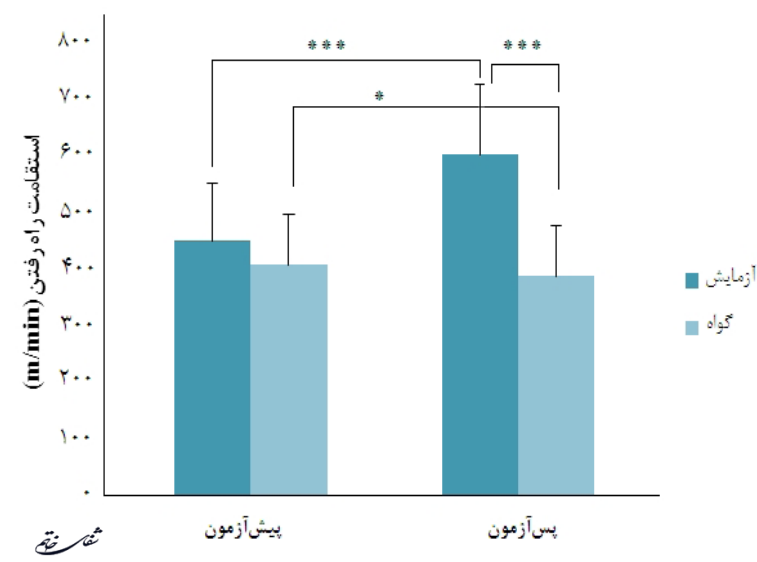

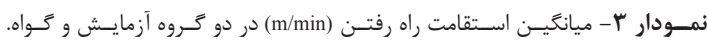

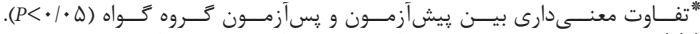

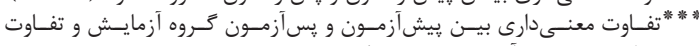

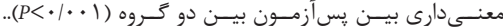

بحث و نتيجه كيرى

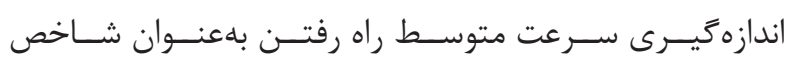

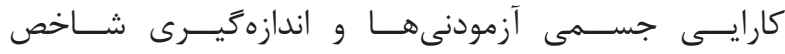

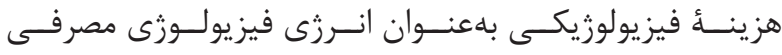

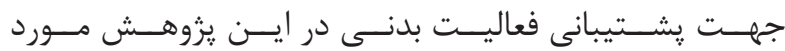

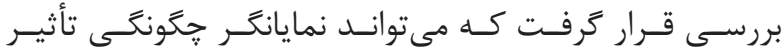

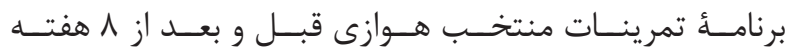

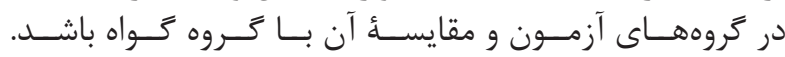

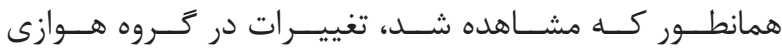

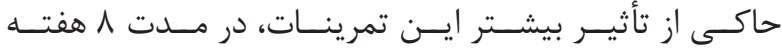

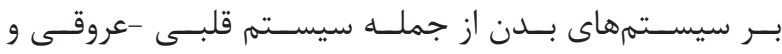

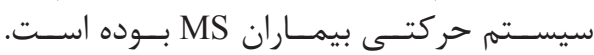

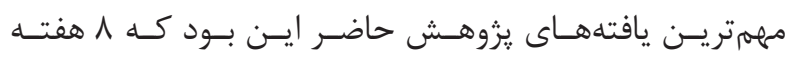

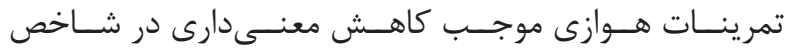

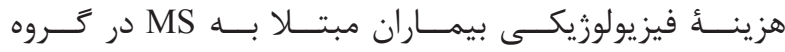

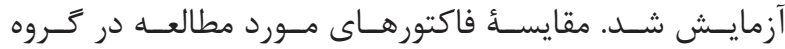

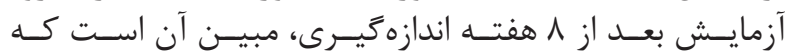

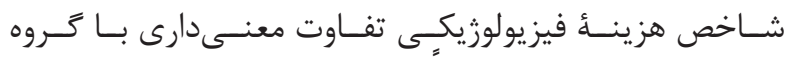

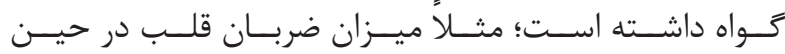

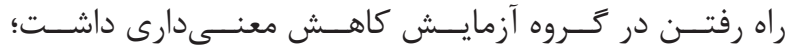

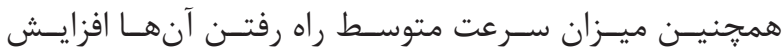
معنسى دارى را نشــان داد.

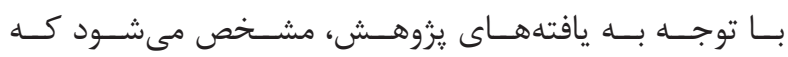

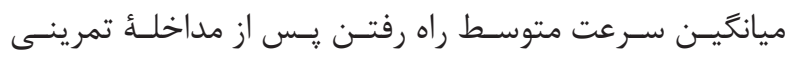

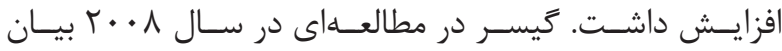

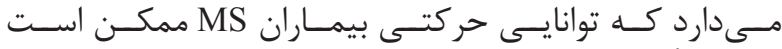

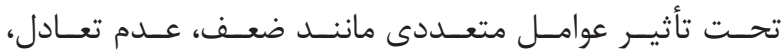

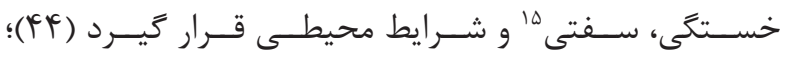

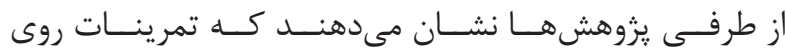

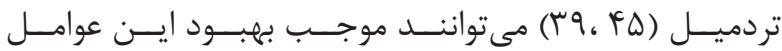




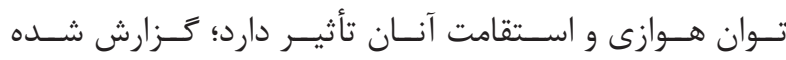

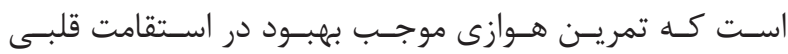

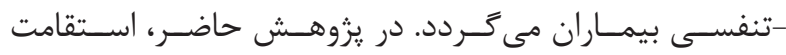

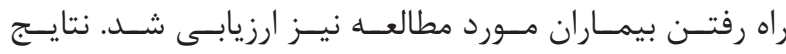

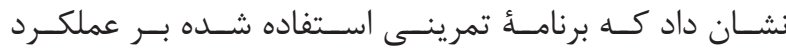

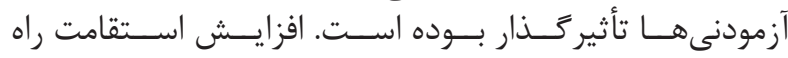

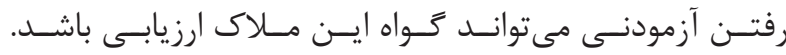

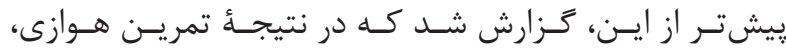

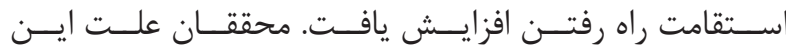

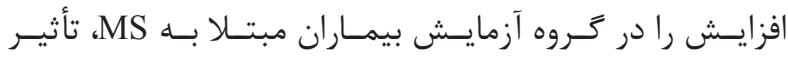

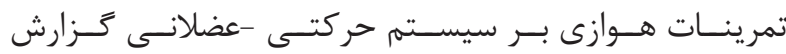

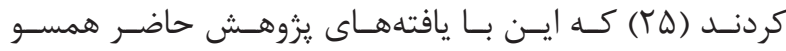

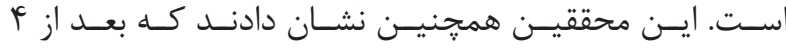

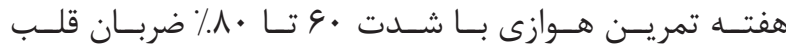

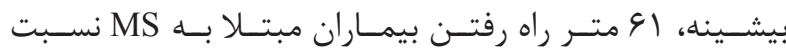

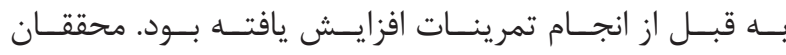

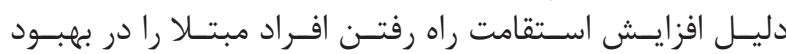

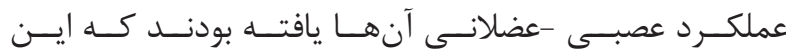

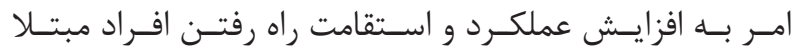

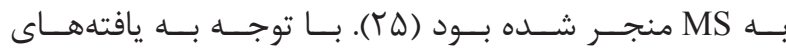

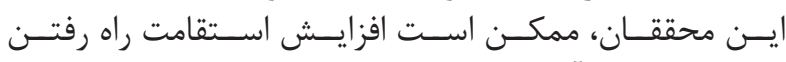

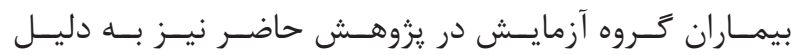

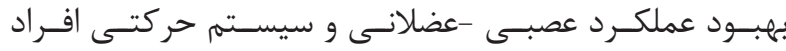

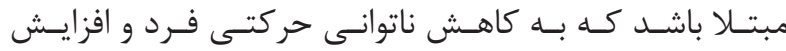

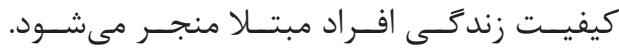

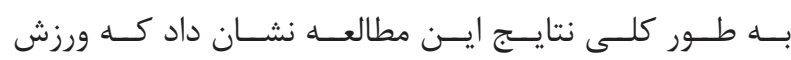

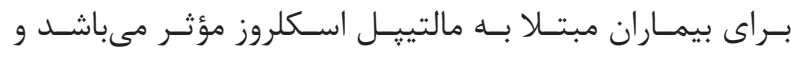

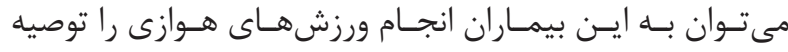

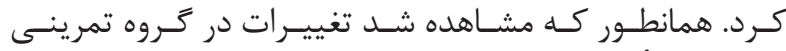

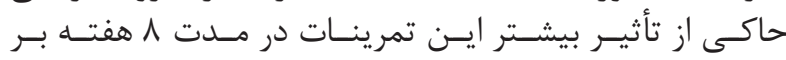

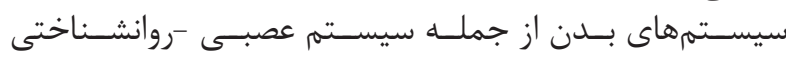

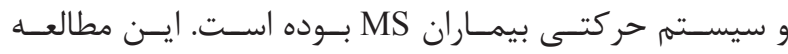

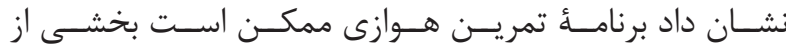

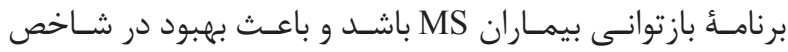

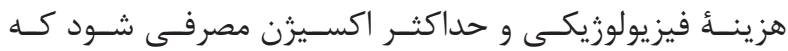

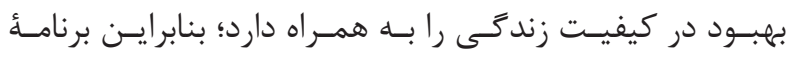

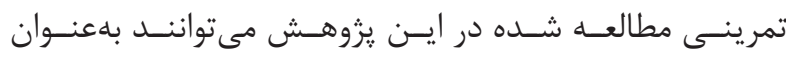

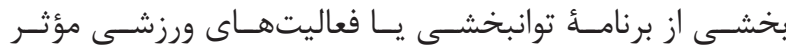

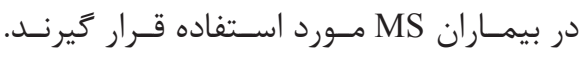

$$
\text { تشكر و قدردانى }
$$

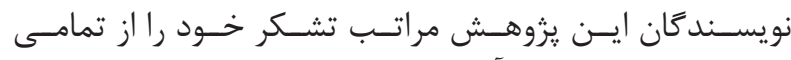

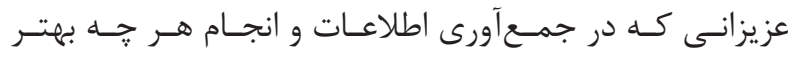

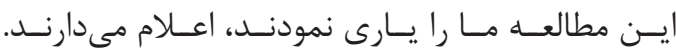

انــرزى بــا همــان مســافت راه رفتـن بهبـود بخشـيد.

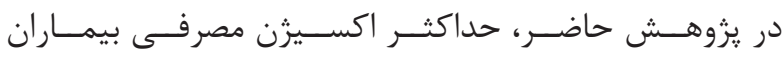

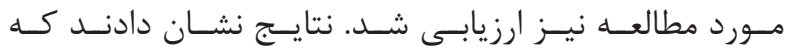

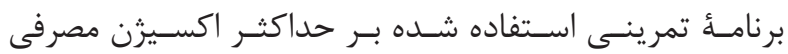

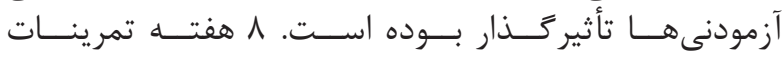

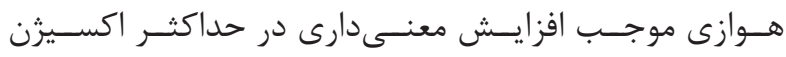

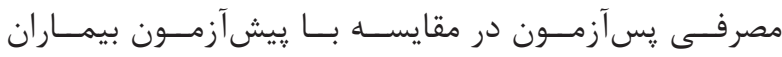

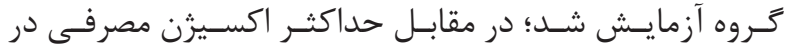

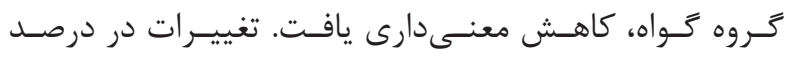

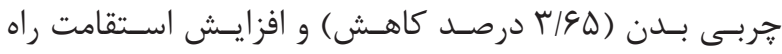

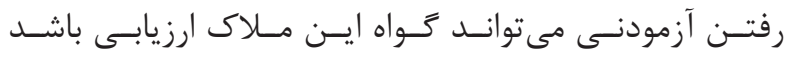

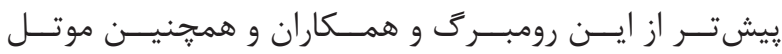

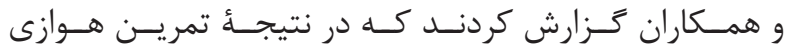

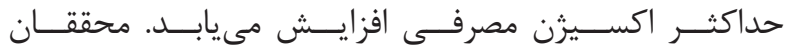

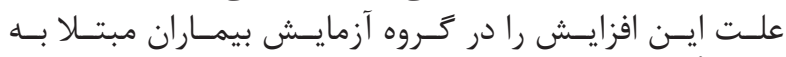
MS

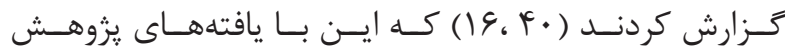

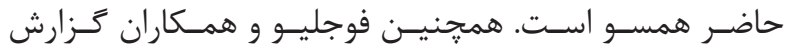

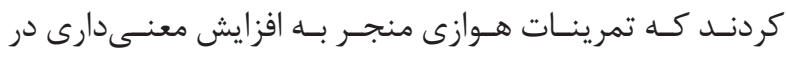

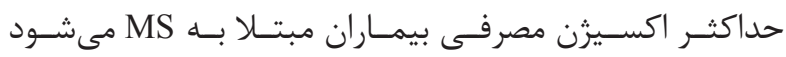

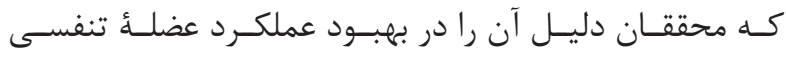

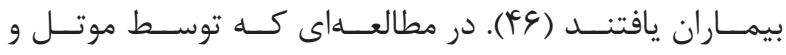

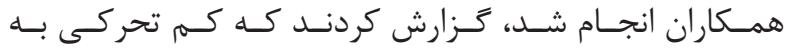

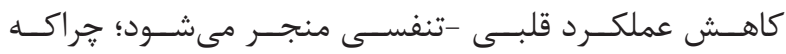

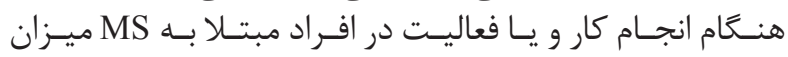

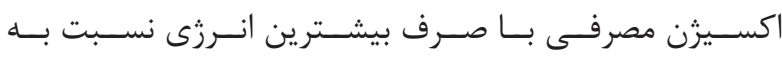

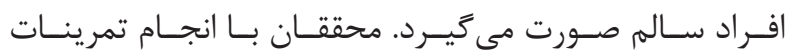

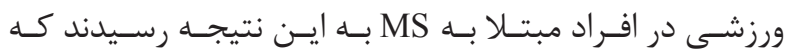

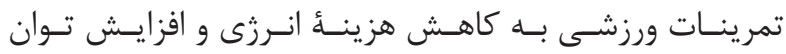

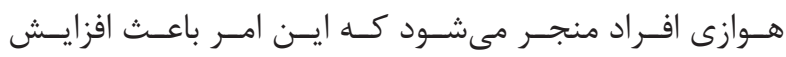

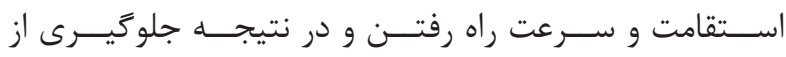

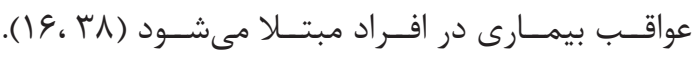

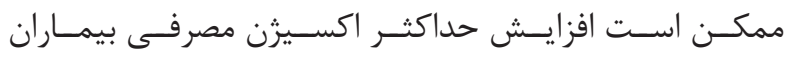

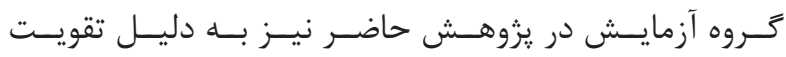

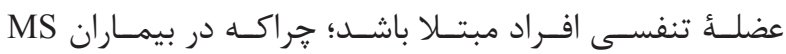

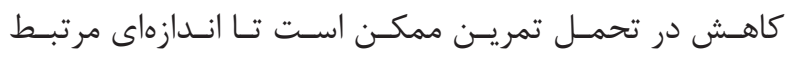

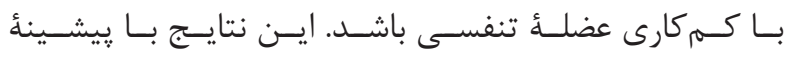

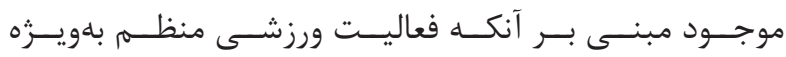

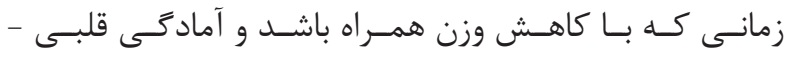

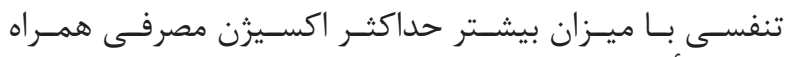

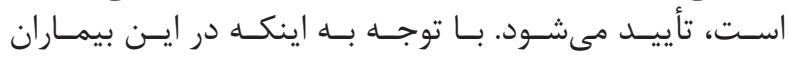

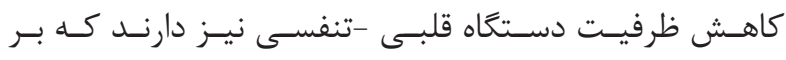


1. Arastoo AA, Ahmadi A, Zahednejad Sh. The comparison of effect of 8 weeks aerobic and yoga training on physiological cost index in multiple sclerosis patients. Sci Med J. 2011; 10(2): 153-62.

2. Eftekhari E, Nikbakht H, Etemadifar M, Rabiee $\mathrm{K}$. Effect of endurance training on aerobic power and quality of life in female patients with multiple sclerosis. Olympic journal. 2008; 16(1): 37-46.

3. Comi G, Leocani L, Rossi P, Colombo B. Physiopathology and treatment of fatigue in multiple sclerosis. J Neurol. 2001; 248(3): 174-9.

4. Etemadifar M, Ashtary F. Diagnosis and treatment of multiple sclerosis. $1^{\text {st }}$ ed. Esfahan. Chahar Bagh publisher. 2002; 13-5.

5. Greenberg DA. Clinical Neurology Aminoff. Translated by Sayed Mostafa Emami and Mohammed Hossein Shoja Moradi. Shabnam Danesh. 2002; 83(13): 123-125.

6. Fernández $\mathrm{O}$, Baumstarck-Barrau K, Simeoni MC, Auquier P, MusiQoL study group. Patient characteristics and determinants of quality of life in an international population with multiple sclerosis: assessment using the musiqol and sf-36 questionnaires. Mult Scler. 2011; 17(10): 1238-49.

7. Miller A, Dishon S. Health-related quality of life in multiple sclerosis: the impact of disability, gender and employment status. Qual life Res. 2006; 15(2): 71-259.

8. Bradly, Daroff, Fenichel, Jankovic. Neurology in clinical practice. $4^{\text {th }}$ ed. Philadelphia: Elsevier, 2004.

9. Schwid SR, Murray TJ. Treating fatigue in patients with MS: one step forward, one step back. Neurology. 2005; 64(7): 1111-2.

10. Carroll L. Does aspirin or modafinil help fatigue in MS? Clinical trials offer mixed results. Neurology. 2005; 5: 38-39.

11. Häuser W, Stallmach A, Kocalevent RD, Rose M, Fliege H. Biopsychosocial predictors of fatigue in quiescent and mild ulcerative colitis. GMS Psychosoc Med. 2005; 2: 1-8.

12. Andreasen AK, Stenager E, Dalgas U. The effect of exercise therapy on fatigue in multiple sclerosis. Mult Scler. 2011; 17(9): 1041-54.

13. Neill J, Belan I, Ried K. Effectiveness of non- pharmacological interventions for fatigue in adults with multiple sclerosis, rheumatoid arthritis, or systemic lupus erythematosus: a systematic review. J Adv Nurs. 2006; 56(6): 617-35.

14. Azimian M. Pschological and bodily in multiple sclerosis. Rehabilitation J. 1999; 3: 43-7.

15. Surakka J, Romberg A, Ruutiainen J, Aunola S, Virtanen A, Karppi SL. Effects of aerobic and strength exercise on motor fatigue in men and women with multiple sclerosis: a randomized controlled trial. Clin Rehabil. 2004; 18(7): 737-46.

16. Motl R, McAuley E, Snook EM, Gliottoni R. Physical activity and quality of life in multiple sclerosis: intermediary roles of disability, fatigue, mood, pain, self-efficacy and social support. Psychol Health Med. 2009; 14(1): 111-24.

17. Rowland LP. Merritt's neurology. $11^{\text {th }}$ ed. Philadelphia: Lippincott Williams \& Wilkins. 2005.

18. Rampello A, Franceschini M, Piepoli M, Antenucci R, Lenti G, Olivieri D. Effect of Aerobic Training on Walking Capacity and Maximal Exercise Tolerance in Patients with Multiple Sclerosis: a Randomized Crossover Controlled Study. Phys Ther. 2007; 87(5): 545-55.

19. Benito-León J. Physical activity in multiple sclerosis: the missing prescription. Neuroepidemiology. 2011; 36: 192-3.

20. Kenedi RM, Paul JP, Hughes J. Disability: Proceedings of a seminar on rehabilitation of the disabled (Strathclyde bioengineering seminars). MacGregor J. Rehabilitation ambulatory monitoring. London: Palgrave Macmillan. 1979; p. 72-159.

21. Broekmans T, Roelants M, Feys P, Alders G, Gijbels D, Hanssen I. Effects of long-term resistance training and simultaneous electro- stimulation on muscle strength and function mobility in multiple sclerosis. Mult Scler. 2011; 17(4): 468-77.

22. Elizabeth HM, Dan MC, Lesley JW, Jennifer LMS, Szu-Yun L, Frank Z. Ratings of Perceived Exertion during Aerobic Exercise in Multiple Sclerosis. Arch Phys Med Rehabil. 2008; 89(8): 1570-4.

23. Robert W, Myla D, Ralph HB. Walking impairment in patients with multiple sclerosis: exercise training as a treatment option. Neuropsychiatr Dis Treat. 2010; 6(16): 767-74. 


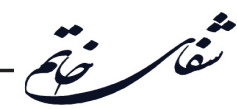

24. Snook EM, Motl RW. Effect of exercise training on walking mobility in multiple sclerosis a meta-analysis. Neurorehabil Neural Repair. 2009; 23(2): 16-108.

25. Kileff J. Aerobic Exercise for People with Multiple Sclerosis. 2004, 8(2): 6-7.

26. Kargarfard M, Etemadifar M, Asfarjani F, Mehrabi M, Kordavani L. Changes in quality of life and fatigue in women with multiple sclerosis after 8 weeks of aquatic exercise training. Journal of Fundamentals of Mental Health. 2010; 12(3): 62-735.

27. Nicole S, Clare M. The impact of regular physical activity on fatigue, depression and quality of life in persons with multiple sclerosis. Health Qual Life Outcomes. 2009; 7(68): 1-10.

28. White LJ, Dressendorfer RH. Exercise and multiple sclerosis. Sports Med. 2004; 34: 1077-100.

29. Petajan JH, Gappmaier E, White AT, Spencer MK, Mino L, Hicks RW. Impact of aerobic training on fitness and quality of life in multiple sclerosis. Am Neurol. 1996; 39(4): 241-3.

30. William A. Application of exercise principles to routine work \& recreational activities in: diagnosis \& prescription of exercise. Protocol for the Chester Step Test. 2011; (23): 240-4.

31. Dalgas U, Stenager E, Jakobsen J, Petersen T, Hansen HJ, Knudsen C. Resistance training improves muscle strength and functional capacity in multiple sclerosis. Neurology. 2009; 73: 1478-84.

32. Chung LH. Muscle weakness in persons with multiple sclerosis. PhD Thesis. University of Massachusetts. 2010 .

33. Vanage, S. M. Gilbertson, KK. Mathiowetz V. Effects of an energy conservation course on fatigue impact for persons with progressive multiple sclerosis. Am J Occup Ther. 2003; 57: 315-23.

34. Yousefvand Z, Parnow A, Ahsan B, Fayazi B. Effect of 8-week resistance training on muscle strength and functional capacity in women with multiple sclerosis (MS). Sabzevar Sport and Biomotor Sciences Journal. 2013; 3(2): 71-82.

35. Savci S, Inal-Ince D, Arikan H, Guclu-Gunduz A, Cetisli-Korkmaz N, Armutlu K. Six-minute walk distance as a measure of functional exercise capacity in multiple sclerosis. Disabil Rehabil. 2005; 27(22): 1365-71.

36. Ozakbas S, Ormeci B, Idiman E. Utilization of the multiple sclerosis functional composite in followup: relationship to disease phenotype, disability and treatment strategies. J Neurol Sci. 2005; 232(1-2): 65-9.
37. Olgiati R, Burgunder JM, Mumenthaler $\mathrm{M}$. Increased energy cost of walking in multiple sclerosis: effect of spasticity, ataxia, and weakness. Arch Phys Med Rehabil. 1988; 69(10): 846-9.

38. Motl RW, Goldman M. Physical inactivity, neurological disability, and cardiorespiratory fitness in multiple sclerosis. Acta Neurol Scand. 2011; 123: 98-104.

39. Newman MA, Dawes H, van den Berg M, Wade DT, Burridge J, Izadi H. Can aerobic treadmill training reduce the effort of walking and fatigue in people with multiple sclerosis: a pilot study. Mult Scler. 2007; 13: 113-9.

40. Romberg A, Virtanen A, Ruutiainen J, Aunola S, Karppi SL, Vaara M. Effect of a 6-month exercise program on patient with multiple sclerosis. Neurology. 2004; 63(11): 2034-8.

41. Ahmadi A, Nikbakh M, Arastoo A, Habibi AH. The effects of a yoga intervention on balance, speed and endurance of walking, fatigue and quality of life in people with multiple sclerosis. Journal of Human Kinetics. 2010; 23: 71-8.

42. Fayazi B, Parnow A, Ahsan B, Yousefvand Z. The effect aerobic training on functional capacity and percent body fat in women with moderate multiple sclerosis (MS). AMUJ. 2013; 16(70): 87-97.

43. Heather A, Paul C. Effects of high-intensity resistance training on strength, mobility, balance, and fatigue in individuals with multiple sclerosis: a randomized controlled trial. J Neurol Phys Ther. 2011; 35: $2-10$.

44. Giesser B, Beres-Jones J, Budovitch A, Herlihy E, Harkema S. Loco motor training using body weight support on a treadmill improves mobility in persons with multiple sclerosis. Mult Scler. 2008; 13(2): 31-224.

45. Giesser BS. Exercise and gait retraining in persons with multiple sclerosis. US Neurol. 2007; 2: 37-9.

46. Foglio K, Clini E, Vitacca M, Marangoni S, Bonomelli M, Ambrosino N. Respiratory muscle functions and exercise capacity in multiple sclerosis. ERS Journals Ltd. 1994; 7: 23-8.

47. Danielsson A. Studies on energy expenditure in walking after stroke. Gothenburg: Institute of Neuroscience and Physiology/Rehabilitation Medicine, University of Gothenburg. 2008; 1-47.

48. Waters RL, Mulroy S. The energy expenditure of normal and pathologic gait. Gait Posture.1999; 9(3): 207-31. 